\title{
Endoscopic versus nonendoscopic surgery for resection of pituitary adenomas: a national database study
}

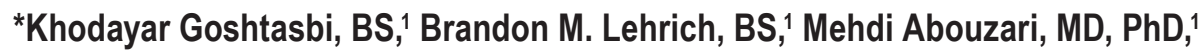 \\ Arash Abiri, BS, ${ }^{1}$ Jack Birkenbeuel, BS, ${ }^{1}$ Ming-Ying Lan, MD, PhD, ${ }^{2}$ Wei-Hsin Wang, MD, ${ }^{3}$ \\ Gilbert Cadena, MD, ${ }^{4}$ Frank P. K. Hsu, MD, PhD, ${ }^{4}$ and Edward C. Kuan, MD, MBA ${ }^{1}$
}

\begin{abstract}
${ }^{1}$ Department of Otolaryngology-Head and Neck Surgery, University of California, Irvine, California; ${ }^{2}$ Department of Otorhinolaryngology-Head and Neck Surgery, Taipei Veterans General Hospital, Taipei, Taiwan; ${ }^{3}$ Department of Neurosurgery, Taipei Veterans General Hospital, Taipei, Taiwan; and ${ }^{4}$ Department of Neurological Surgery, University of California, Irvine, California
\end{abstract}

OBJECTIVE For symptomatic nonsecreting pituitary adenomas (PAs), resection remains a critical option for treatment. In this study, the authors used a large-population national database to compare endoscopic surgery (ES) to nonendoscopic surgery (NES) for the surgical management of PA.

METHODS The National Cancer Database was queried for all patients diagnosed with histologically confirmed PA who underwent resection between 2010 and 2016 in which the surgical approach was specified. Due to database limitations, microsurgery and craniotomy were both categorized as NES.

RESULTS Of 30,488 identified patients, 16,373 (53.7\%) underwent ES and 14,115 (46.3\%) underwent NES. There was a significant increase in the use of ES over time (OR 1.16, $p<0.01)$. Furthermore, there was a significant temporal increase in ES approach for tumors $\geq 2 \mathrm{~cm}$ (OR 1.17, $p<0.01)$. Compared to NES, patients who underwent ES were younger $(p=0.01)$, were treated at academic centers $(p<0.01)$, lived a greater distance from their treatment site $(p<$ 0.01 ), had smaller tumors $(p<0.01)$, had greater medical comorbidity burden $(p=0.04)$, had private insurance $(p<0.01)$, and had a higher household income $(p<0.01)$. After propensity score matching to control for age, tumor size, Charlson/ Deyo score, and type of treatment center, patients who underwent ES had a shorter length of hospital stay (LOS) (3.9 \pm 4.9 days vs $4.3 \pm 5.4$ days, $p<0.01)$, although rates of gross-total resection (GTR; $p=0.34)$, adjuvant radiotherapy $(p=$ 0.41 ), and 90-day mortality ( $p=0.45)$ were similar. On multivariate logistic regression, African American race (OR 0.85, $p<0.01$ ) and tumor size $\geq 2 \mathrm{~cm}$ (OR 0.89, $p=0.01$ ) were negative predictors of receiving ES, whereas diagnosis in more recent years (OR 1.16, $p<0.01)$, greater Charlson/Deyo score (OR 1.10, $p=0.01)$, receiving treatment at an academic institution (OR 1.67, $p<0.01$ ) or at a treatment site $\geq 20$ miles away (OR 1.17, $p<0.01)$, having private insurance (OR $1.09, p=0.01$ ), and having a higher household income (OR 1.11, $p=0.01$ ) were predictive of receiving ES. Compared to the ES cohort, patients who started with ES and converted to NES $(n=293)$ had a higher ratio of nonwhite race $(p<$ $0.01)$, uninsured insurance status $(p<0.01)$, longer LOS $(p<0.01)$, and higher rates of GTR $(p=0.04)$.

CONCLUSIONS There is an increasing trend toward ES for PA resection including its use for larger tumors. Although ES may result in shorter LOS compared to NES, rates of GTR, need for adjuvant therapy, and short-term mortality may be similar. Factors such as tumor size, insurance status, facility type, income, race, and existing comorbidities may predict receiving ES.

https://thejns.org/doi/abs/10.3171/2020.1.JNS193062

KEYWORDS pituitary adenoma; endoscopy; microscopic surgery; transsphenoidal; NCDB; National Cancer Database; surgical outcome; pituitary surgery; surgical technique

$\mathrm{S}$ TUDIES of various geographic regions have suggested a 1 in 860-2690 prevalence for pituitary adenomas (PAs), where $14 \%-54 \%$ of patients could present with clinically nonfunctioning tumors. ${ }^{30}$ For symptomatic tumors, resection remains a critical treatment option, with high rates of tumor control. Inspired by the work of Jules Hardy in the $1960 \mathrm{~s},{ }^{19}$ microscopic transsphenoidal surgery has been a mainstay of surgical treatment. More recently, the first endoscopic pituitary surgery was reported by Jankowski et al. in $1992 .{ }^{20}$ Since then, emerging interest

ABBREVIATIONS $\mathrm{C} / \mathrm{D}=$ Charlson/Deyo; $\mathrm{ES}$ = endoscopic surgery; GTR = gross-total resection; LOS = length of hospital stay; NCDB = National Cancer Database; NES

= nonendoscopic surgery; $\mathrm{PA}=$ pituitary adenoma.

SUBMITTED November 11, 2019. ACCEPTED January 2, 2020.

INCLUDE WHEN CITING Published online March 13, 2020; DOI: 10.3171/2020.1.JNS193062.

${ }^{*}$ K.G. and B.M.L. contributed equally to this work. 
in endoscopic resection has led to numerous institutional studies, ${ }^{1,2,11,43}$ along with various systematic reviews and meta-analyses ${ }^{3,4,18}$ comparing endoscopic surgery (ES) and nonendoscopic surgery (NES) in PA resection. Gradual adoption of ES can be attributed to several factors such as increased comfort with endoscopic techniques, decreased morbidity, broader visualization via dynamic endoscopy, and lower postoperative complication rates. ${ }^{3,23,24}$ On the other hand, ES is associated with a higher initial learning curve due to its 2D field of view. ${ }^{31,39}$ There are also contradictory data in the literature regarding certain postoperative outcomes, such as ability to achieve gross-total resection (GTR). ${ }^{3,16,24}$ Furthermore, reports of readmission and reoperation have been limited to single-institution studies or small meta-analyses as opposed to investigating large-population national databases..$^{10,15,45}$ Therefore, there remain continuous discussions regarding the benefits and drawbacks of either approach depending on the patient population, desired results, and available resources.

Despite the nationwide shift toward ES at the expense of NES, ${ }^{6,35}$ the existing literature also does not provide an in-depth exploration of the demographic and preclinical factors such as income, insurance status, race, distance to provider, and medical comorbidities in predicting ES. As such, this study uses one of the largest national tumor databases, which to our knowledge has not been used for assessing surgical treatment of PA, in order to compare ES and NES regarding clinical and demographic/socioeconomic presentations, surgical outcomes, temporal trends, and factors predictive of patients undergoing ES.

\section{Methods}

This study was exempt from institutional review board approval because of the de-identified nature of subject information reported by this publicly available national database. The National Cancer Database (NCDB) is a national registry sponsored by the American Cancer Society and the American College of Surgeons that collects data from more than 1500 hospitals and encompasses most of the new cancer diagnoses in the nation. ${ }^{8}$ The NCDB was queried for all patients diagnosed with histologically confirmed PA from 2004 to 2016. However, because the surgical approach (ES vs NES) was only specified starting in 2010 for the NCDB, patients treated before 2010 were excluded. Primary site topographical code C75.1 (pituitary gland) and International Classification of Diseases for Oncology, Third Edition histology codes for PA (8140, $8202,8260,8270,8271,8272,8280,8281,8290,8300$ ) were used. The NCDB does not differentiate between microscopic transsphenoidal surgery and open approaches (e.g., craniotomies); thus, both were combined as NES. Patients who underwent palliative treatment were excluded. Among the NES cohort, a minority of patients were coded as "endoscopic converted to open," which is meant to capture patients who started with a planned ES that was then converted to an open approach intraoperatively as part of the same operation. Extracted data consisted of patient demographics, tumor characteristics, diagnosis and treatment protocols, unplanned reoperations, and 30and 90-day survival outcomes. Tumor size was coded as a
2D value representing the largest diameter of the primary tumor (in millimeters). In analyzing tumor size, tumors reported as $<3 \mathrm{~mm}$ or $>10.5 \mathrm{~cm}$ were excluded to avoid potential errors in reporting. The Charlson/Deyo (C/D) comorbidity index score, which is an indication of the patient's overall health status due to existing comorbidities, was categorized as patients with no comorbidities (score 0 ) versus those with comorbidities (score $\geq 1$ ).

Propensity score matching was performed to adjust for potential baseline confounding characteristics in the data and to balance the ES and NES cohorts to simulate randomization. This analysis was performed using the statistical programming language $\mathrm{R}$ (version 3.6.1; The R Foundation for Statistical Computing, Vienna, Austria; https:// cran.r-project.org) via RStudio (version 1.2.1335). We used the nearest-neighbor matching method, in which the "MatchIt" package in $\mathrm{R}$ with 1:1 matching and a caliper of 0.01 was used. Variables that were matched included age, tumor size, C/D score, and treatment facility type. All variables were then statistically compared between the 2 cohorts to confirm the matching algorithm. All other statistical analyses were performed using PASW Statistics 18.0 software (SPSS, Inc.) with a threshold of $p<0.05$ for statistical significance. Independent-samples t-test and chi-square tests were used to compare mean differences in continuous and categorical variables, respectively. Clinical and demographic factors predictive of receiving ES were determined using binary logistic regression, with factors significant on univariate analysis combined and adjusted through multivariate analysis to determine independent predictors for receiving ES.

\section{Results}

There were 30,488 surgically treated patients with PA, consisting of $47.9 \%$ females with a mean age of $53.0 \pm$ 15.8 years and a mean tumor size of $24.4 \pm 12.8 \mathrm{~mm}$. Among this PA cohort, 16,373 (53.7\%) received ES and $14,115(46.3 \%)$ underwent NES-the demographic and clinical presentations of the 2 cohorts are compared in Table 1. This demonstrated that younger age $(p=0.01)$, smaller tumor size $(p<0.01)$, Caucasian race $(p<0.01)$, greater medical comorbidity burden $(p=0.04)$, private insurance $(\mathrm{p}<0.01)$, treatment at an academic center ( $\mathrm{p}$ $<0.01)$, higher household income $(\mathrm{p}<0.01)$, and living a greater distance from the treatment site $(\mathrm{p}<0.01)$ were factors more frequently encountered with receiving ES. Temporal analysis of the years 2010-2016 demonstrated a significant increase in ES approach over time $\left(\mathrm{R}^{2}=0.915\right.$, $\mathrm{p}<0.01$ ) (Fig. 1).

Univariate logistic regression demonstrated that, compared to the year 2010, patients diagnosed in succeeding years were more likely to receive ES compared to NES. The findings were as follows: it was 1.52 times more likely (OR $1.520,95 \%$ CI $1.394-1.658, \mathrm{p}<0.01$ ) for those diagnosed in 2011; 1.72 times (OR 1.722, 95\% CI 1.580-1.877, p $<0.01$ ) for those diagnosed in 2012; 2.00 times (OR 1.998, $95 \%$ CI 1.834-2.177, $\mathrm{p}<0.01$ ) for those diagnosed in 2013; 2.31 times (OR 2.310, 95\% CI 2.120-2.517, p < 0.01) for those diagnosed in 2014; 2.39 times (OR 2.385, 95\% CI $2.189-2.600, \mathrm{p}<0.01$ ) for those diagnosed in 2015; and 
TABLE 1. Clinical and demographic factors comparing ES to NES for PA

\begin{tabular}{|c|c|c|c|c|}
\hline Covariate & Total, $\mathrm{N}=30,488$ & $E S, n=16,373$ & NES, $n=14,115$ & $p$ Value \\
\hline Age in yrs, mean \pm SD & $53.0 \pm 15.8$ & $52.8 \pm 15.8$ & $53.3 \pm 15.8$ & 0.01 \\
\hline Sex, no. female $(\%)$ & $14,602(47.9)$ & $7,863(48.0)$ & $6,739(47.7)$ & 0.63 \\
\hline Race, no. (\%) & & & & $<0.01^{*}$ \\
\hline Caucasian & $22,277(74.1)$ & $12,157(75.3)$ & $10,120(72.8)$ & \\
\hline African American & $5,772(19.2)$ & $2,860(17.7)$ & $2,912(20.9)$ & \\
\hline Other/unknown & $1,999(6.7)$ & $1,121(6.9)$ & $878(6.3)$ & \\
\hline Tumor size in $\mathrm{mm}$, mean $\pm \mathrm{SD}$ & $24.4 \pm 12.8$ & $23.8 \pm 12.5$ & $25.2 \pm 13.0$ & $<0.01^{*}$ \\
\hline C/D index, no. $(\%)$ & & & & 0.04 \\
\hline 0 & $22,943(75.3)$ & $12,245(74.8)$ & $10,698(75.8)$ & \\
\hline$\geq 1$ & $7,545(24.7)$ & $4,128(25.2)$ & $3,417(24.4)$ & \\
\hline Facility type, no. (\%) & & & & $<0.01^{*}$ \\
\hline Nonacademic & $8,772(36.6)$ & $3,986(31.1)$ & $4,786(43.0)$ & \\
\hline Academic & $15,186(63.4)$ & $8,832(68.9)$ & $6,354(57.0)$ & \\
\hline Insurance status, no. (\%) & & & & $<0.01^{*}$ \\
\hline Private & $17,335(58.0)$ & $9,580(59.3)$ & $7,755(56.6)$ & \\
\hline Government & $11,085(37.1)$ & $5,952(36.8)$ & $5,133(37.4)$ & \\
\hline Uninsured & $1,451(4.9)$ & $626(3.9)$ & $825(6.0)$ & \\
\hline Income, no. (\%) & & & & $<0.01^{*}$ \\
\hline$<\$ 48,000$ & $12,068(39.7)$ & $6,207(38.0)$ & $5,861(41.6)$ & \\
\hline$\geq \$ 48,000$ & $18,367(60.3)$ & $10,143(62.0)$ & $8,224(58.4)$ & \\
\hline ZIP code, residents w/o HSD, no. (\%) & & & & $<0.01^{*}$ \\
\hline$<13 \%$ & $14,030(46.1)$ & $7,238(44.3)$ & $6,792(48.2)$ & \\
\hline$\geq 13 \%$ & $16,418(53.9)$ & $9,117(55.7)$ & $7,301(51.8)$ & \\
\hline Urban/rural population, no. (\%) & & & & 0.82 \\
\hline Metro counties & $25,271(85.0)$ & $13,569(84.9)$ & $11,702(85.0)$ & \\
\hline Urban or rural counties & $4,462(15.0)$ & $2,404(15.1)$ & $2,058(15.0)$ & \\
\hline Distance from patient to provider in miles, mean \pm SD & $55.4 \pm 145.3$ & $61.6 \pm 157.1$ & $48.1 \pm 129.9$ & $<0.01^{*}$ \\
\hline
\end{tabular}

HSD = high school diploma.

All percentages reflect denominators encompassing known or reported values, which do not always equate to the total cohort's number. The $p$ values indicate chi-square test for categorical variables or independent t-test for continuous variables in comparing ES to NES. Boldface type indicates statistical significance.

${ }^{*}$ Denotes $p<0.001$; all p values expressed only to 2 decimal places.

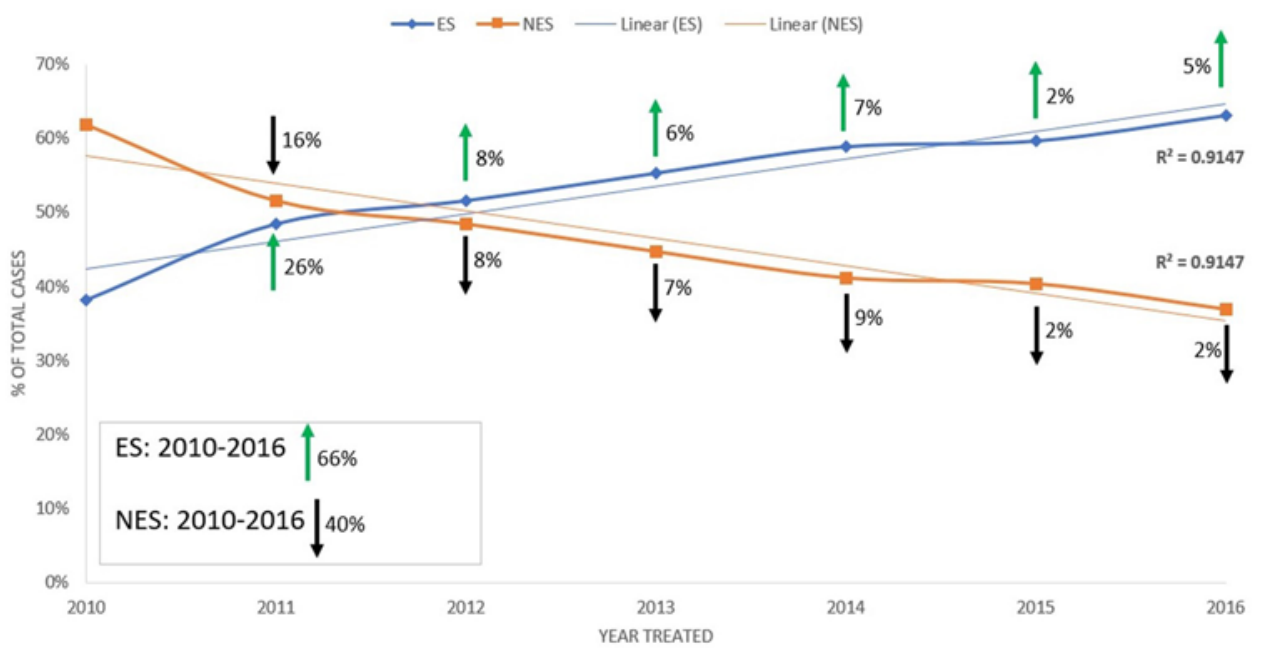

FIG. 1. Temporal trends of type of surgical approach for resection of PA. There has been a significant increase in the adoption of ES over time ( $p<0.01)$, with a clear $66 \%$ increase in the use of ES from 2010 to 2016 and a $40 \%$ decrease in the use of NES during this timeline. Figure is available in color online only. 
TABLE 2. Clinical outcomes comparing ES to NES for PA

\begin{tabular}{lccccc}
\hline \multicolumn{1}{c}{ Covariate } & Total, $\mathrm{N}=30,488$ & $\mathrm{ES}, \mathrm{n}=16,373$ & $\mathrm{NES}, \mathrm{n}=14,115$ & $\mathrm{OR}, 95 \% \mathrm{Cl}$ & $\mathrm{p} \mathrm{Value}$ \\
\hline GTR, no. (\%) & $10,842(75.5)$ & $5,493(73.9)$ & $5,349(77.2)$ & $0.839,0.778-0.906$ & $<0.01^{*}$ \\
\hline LOS in days, mean \pm SD & $3.9 \pm 5.3$ & $3.7 \pm 4.6$ & $4.3 \pm 6.1$ & $0.975,0.970-0.980$ & $<0.01^{*}$ \\
\hline Readmission w/in 30 days of surgical discharge, no. (\%) & $1,210(4.0)$ & $621(3.8)$ & $589(4.2)$ & $0.904,0.806-1.014$ & 0.09 \\
\hline Adjuvant radiation, no. (\%) & $1,275(4.2)$ & $577(3.5)$ & $630(4.5)$ & $0.784,0.701-0.877$ & $<0.01^{*}$ \\
\hline 30-day mortality, no. (\%) & $201(0.8)$ & $83(0.6)$ & $118(1.0)$ & $0.642,0.485-0.851$ & $<0.01$ \\
\hline 90-day mortality, no. (\%) & $327(1.3)$ & $137(1.1)$ & $190(1.6)$ & $0.658,0.528-0.821$ & $<0.01^{*}$ \\
\hline
\end{tabular}

All percentages reflect denominators encompassing known or reported values, which do not always equate to the total cohort's number. The $p$ values indicate chisquare test for categorical variables or independent t-test for continuous variables in comparing ES to NES. The OR values are calculated with univariate logistic regression comparing ES to NES use. Boldface type indicates statistical significance.

* Denotes $p<0.001$; all $p$ values expressed only to 2 decimal places.

2.76 times more likely (OR 2.759, 95\% CI 2.528-3.011, p < 0.01 ) for those diagnosed in 2016 to receive ES compared to NES. Univariate logistic regression also demonstrated that, compared to patients diagnosed in 2010-2013, patients diagnosed in 2014-2016 were 1.65 times more likely (OR 1.653, 95\% CI 1.461-1.869, $\mathrm{p}<0.01$ ); 1.59 times more likely (OR 1.586, 95\% CI 1.464-1.718, p < 0.01); and 1.52 times more likely (OR 1.522, 95\% CI 1.404-1.650, p < $0.01)$ to undergo ES in the western, central, and eastern US regions, respectively. Among tumors treated at academic institutions $(\mathrm{n}=15,186)$, univariate logistic regression demonstrated that, compared to those diagnosed between 2010 and $2013(n=8451)$, tumors diagnosed between 2014 and $2016(n=6735)$ were 1.65 times more likely to undergo ES compared to NES (OR 1.652, 95\% CI 1.563-1.745, $\mathrm{p}<0.01$ ); and, among tumors treated at nonacademic institutions $(\mathrm{n}=8772)$, compared to those diagnosed between 2010 and $2013(\mathrm{n}=5024)$, tumors diagnosed between 2014 and $2016(\mathrm{n}=3748)$ were 1.57 times more likely to undergo ES compared to NES (OR 1.568, 95\% CI 1.440-1.707, $\mathrm{p}<0.01)$. The ratio of tumors $\geq 2 \mathrm{~cm}(\mathrm{n}=13,422)$ undergoing ES consisted of $43.7 \%$ in years $2010-2011,53.4 \%$ in years 2012-2013, and 59.7\% in years 2014-2016. Likewise, the ratio of $\geq 4$-cm tumors $(\mathrm{n}=1998)$ undergoing ES consisted of $43.1 \%$ in years $2010-2011,45.6 \%$ in years 2012-2013, and 57.1\% in years 2014-2016. Temporal analysis of the years 2010-2016 demonstrated a significant increase in ES approach over time for tumors $\geq 2 \mathrm{~cm}$ (OR $1.173,95 \%$ CI 1.149-1.197, p < 0.01) and $\geq 4 \mathrm{~cm}$ (OR 1.139, $95 \%$ CI 1.080-1.201, p < 0.01).
In comparing the postoperative outcomes for ES and NES, ES was associated with shorter postoperative length of hospital stay (LOS; $p<0.01$ ), lower rates of GTR ( $p$ $<0.01)$, lower 30-day $(\mathrm{p}<0.01)$ and 90-day $(\mathrm{p}<0.01)$ mortality rates, and lower rates of adjuvant radiotherapy $(\mathrm{p}<0.01)$ (Table 2). After we performed propensity score matching to control for age, tumor size, C/D score, and treatment facility type, the resulting cohort of 5250 subjects comprised 2 groups (ES, 2625 patients; NES, 2625 patients) who had similar rates of being treated at an academic center $(64.7 \%$ vs $65.8 \%, \mathrm{p}=0.40)$; age $\geq 65$ years $(34.6 \%$ vs $33.0 \%, \mathrm{p}=0.23) ; \mathrm{C} / \mathrm{D}$ score $\geq 1(25.8 \%$ vs $27.4 \%, \mathrm{p}=0.17)$; and tumor size $\geq 2 \mathrm{~cm}(71.7 \%$ vs $71.5 \%$, $\mathrm{p}=0.83$ ). The outcomes of the 2 groups are compared in Table 3, which demonstrates similar rates of GTR, 30- and 90-day mortality, readmission, and need for postoperative adjuvant radiation. However, following propensity score matching, LOS following ES was significantly lower than that after NES ( $3.9 \pm 4.9$ days vs $4.3 \pm 5.4$ days, $\mathrm{p}=0.01)$.

When comparing all ES cases $(\mathrm{N}=16,373)$ to those that were started as ES but converted to NES ( $\mathrm{n}=293)$, race $(24.7 \%$ vs $32.3 \%$ nonwhite, $p<0.01)$; insurance $(3.9 \%$ vs $8.0 \%$ uninsured/unknown, $\mathrm{p}<0.01$ ); distance to provider $(61.6 \pm 157.1$ miles vs $40.2 \pm 65.5$ miles, $p=0.02)$; LOS (3.7 \pm 4.6 days vs $4.8 \pm 6.1$ days, $\mathrm{p}<0.01)$; and GTR (73.9\% vs $81.6 \%, \mathrm{p}=0.04)$ were significantly different. On multivariate logistic regression of this cohort's presurgical variables, uninsured/unknown insurance status was the only significant predictor of conversion from ES to NES (OR 2.014, 95\% CI 1.158-3.503, p < 0.01).

TABLE 3. Clinical outcomes comparing ES to NES for PA in propensity score-matched cohorts

\begin{tabular}{lccccc}
\hline \multicolumn{1}{c}{ Covariate } & Total, $\mathrm{N}=5250$ & $\mathrm{ES}, \mathrm{n}=2625$ & $\mathrm{NES}, \mathrm{n}=2625$ & $\mathrm{OR}, 95 \% \mathrm{Cl}$ & $\mathrm{p}$ Value \\
\hline GTR, no. (\%) & $3,759(71.6)$ & $1,864(71.0)$ & $1,895(72.2)$ & $0.944,0.837-1.064$ & 0.34 \\
\hline LOS in days, mean \pm SD & $4.1 \pm 5.2$ & $3.9 \pm 4.9$ & $4.3 \pm 5.4$ & $0.986,0.975-0.997$ & 0.01 \\
\hline Readmission w/in 30 days of surgical discharge, no. (\%) & $220(4.2)$ & $100(3.8)$ & $120(4.6)$ & $0.827,0.630-1.084$ & 0.17 \\
\hline Adjuvant radiation, no. (\%) & $300(5.7)$ & $157(6.0)$ & $143(5.4)$ & $1.104,0.874-1.394$ & 0.41 \\
\hline 30-day mortality, no. (\%) & $36(0.7)$ & $19(0.7)$ & $17(0.6)$ & $1.119,0.580-2.157$ & 0.74 \\
\hline 90-day mortality, no. (\%) & $64(1.2)$ & $29(1.1)$ & $35(1.3)$ & $0.827,0.504-1.356$ & 0.45 \\
\hline
\end{tabular}

The $p$ values indicate chi-square test for categorical variables or independent t-test for continuous variables in comparing ES to NES. The OR values are calculated with univariate logistic regression comparing ES to NES use. Boldface type indicates statistical significance. 
TABLE 4. Clinical and demographic factors predictive of receiving ES $(n=16,373)$ in comparison to NES $(n=14,115)$ for PA

\begin{tabular}{|c|c|c|c|c|}
\hline Covariate & Univariate OR $(95 \% \mathrm{Cl})$ & $p$ Value & Multivariate OR $(95 \% \mathrm{Cl})$ & p Value \\
\hline \multicolumn{5}{|l|}{ Age, yrs } \\
\hline$<65$ & 1 [Reference] & \multicolumn{3}{|c|}{1 [Reference] } \\
\hline$\geq 65$ & $0.952(0.905-1.002)$ & 0.06 & NS & \\
\hline \multicolumn{5}{|l|}{ Sex } \\
\hline Male & 1 [Reference] & \multicolumn{3}{|c|}{1 [Reference] } \\
\hline Female & $1.011(0.967-1.058)$ & 0.63 & NS & \\
\hline \multicolumn{5}{|l|}{ Race } \\
\hline Caucasian & 1 [Reference] & \multicolumn{3}{|c|}{1 [Reference] } \\
\hline African American & $0.818(0.772-0.866)$ & $<0.01^{*}$ & $0.852(0.783-0.927)$ & $<0.01^{*}$ \\
\hline Other & $1.063(0.969-1.165)$ & 0.20 & $1.030(0.899-1.181)$ & 0.68 \\
\hline Yr of diagnosis, continuous & $1.163(1.150-1.177)$ & $<0.01^{*}$ & $1.162(1.141-1.185)$ & $<0.01$ \\
\hline \multicolumn{5}{|l|}{ Tumor size, $\mathrm{cm}$} \\
\hline$<2$ & 1 [Reference] & \multicolumn{3}{|c|}{1 [Reference] } \\
\hline$\geq 2$ & $0.838(0.790-0.888)$ & $<0.01^{*}$ & $0.885(0.825-0.950)$ & $<0.01$ \\
\hline \multicolumn{5}{|l|}{$C / D$ index } \\
\hline 0 & 1 [Reference] & \multicolumn{3}{|c|}{1 [Reference] } \\
\hline$\geq 1$ & 1.055 (1.002-1.112) & 0.04 & $1.101(1.025-1.184)$ & 0.01 \\
\hline \multicolumn{5}{|l|}{ Facility type } \\
\hline Nonacademic & 1 [Reference] & \multicolumn{3}{|c|}{1 [Reference] } \\
\hline Academic & $1.669(1.583-1.760)$ & $<0.01^{*}$ & $1.673(1.564-1.789)$ & $<0.01^{*}$ \\
\hline \multicolumn{5}{|l|}{ Insurance status } \\
\hline Government & 1 [Reference] & & & \\
\hline Private & $1.065(1.016-1.118)$ & 0.01 & $1.094(1.022-1.170)$ & 0.01 \\
\hline Uninsured & $0.654(0.586-0.731)$ & $<0.01^{*}$ & $0.677(0.574-0.798)$ & $<0.01^{*}$ \\
\hline \multicolumn{5}{|l|}{ Income } \\
\hline$<\$ 48,000$ & 1 [Reference] & \multicolumn{3}{|c|}{1 [Reference] } \\
\hline$\geq \$ 48,000$ & $1.165(1.112-1.219)$ & $<0.01^{*}$ & $1.112(1.028-1.203)$ & 0.01 \\
\hline \multicolumn{5}{|l|}{ ZIP code, residents w/o HSD } \\
\hline$<13 \%$ & 1 [Reference] & \multicolumn{3}{|c|}{1 [Reference] } \\
\hline$\geq 13 \%$ & $0.853(0.816-0.893)$ & $<0.01^{*}$ & $1.014(0.939-1.095)$ & 0.72 \\
\hline \multicolumn{5}{|l|}{ Urban/rural population } \\
\hline Metro counties & 1 [Reference] & \multicolumn{3}{|c|}{1 [Reference] } \\
\hline Urban or rural counties & $0.993(0.931-1.058)$ & 0.82 & NS & \\
\hline \multicolumn{5}{|l|}{ Distance to provider, miles } \\
\hline$<20$ & 1 [Reference] & \multicolumn{3}{|c|}{1 [Reference] } \\
\hline$\geq 20$ & $1.305(1.248-1.366)$ & $<0.01^{*}$ & $1.167(1.091-1.249)$ & $<0.01^{*}$ \\
\hline
\end{tabular}

NS = not significant.

Boldface type indicates statistical significance.

* Denotes $p<0.001$; all $p$ values expressed only to 2 decimal places.

Univariate and multivariate logistic regression were performed to elicit factors predictive of receiving ES (Table 4). On multivariate regression, patients of African American race (OR 0.852, 95\% CI 0.783-0.927, p < 0.01) or who had a tumor size $\geq 2 \mathrm{~cm}$ (OR 0.885 , 95\% CI 0.825 $0.950, \mathrm{p}<0.01)$ were less likely to receive ES, whereas patients diagnosed at a more recent year (OR 1.162, 95\% CI 1.141-1.185, $\mathrm{p}<0.01$ ); with a C/D score $\geq 1$ (OR 1.101, 95\% CI 1.025-1.184, $\mathrm{p}=0.01)$; treated at an academic institution (OR 1.673, 95\% CI 1.564-1.789, $\mathrm{p}<0.01$ ); having private insurance (OR 1.094, 95\% CI 1.022-1.170, $\mathrm{p}=$
0.01 ); having greater household income (OR 1.112,95\% CI $1.028-1.203, \mathrm{p}=0.01$ ); and living $\geq 20$ miles distant from their provider's treatment site (OR 1.167, 95\% CI 1.091$1.249, \mathrm{p}<0.01)$ were more likely to receive ES.

\section{Discussion}

This is the first study to use the NCDB to compare ES and NES rates, predictive factors, and outcomes for PA resection. We demonstrated a positive temporal trend toward ES use, different demographic and clinical presentations 
between the 2 cohorts, similar postoperative outcomes, and the existence of multiple clinical and demographic/ socioeconomic factors predictive of receiving ES. Specifically, ES was more frequently observed in patients with younger age, smaller tumor size, higher number of medical comorbidities, private insurance, greater household income, receiving treatment at an academic center, and residing farther from their treatment site. In our propensity score-matched analysis, we observed similar rates of GTR, need for adjuvant radiotherapy, unplanned readmission, and short-term mortality, yet patients who underwent NES experienced a longer LOS. Last, we found negative predictors of receiving ES to include African American race, larger tumor size, fewer medical comorbidities, diagnosis in less recent years, lower household income, uninsured patients, and receiving treatment at a nonacademic center.

Our NCDB data demonstrated a clear temporal trend toward ES adoption, with recent diagnosis year also being an independent predictor for receiving ES. These findings are corroborated with 2 other studies in which different national database studies were used. Rolston et al. and Asemota et al. analyzed the 2003-2013 Centers for Medicare and Medicaid Services Part B and the 2010-2014 Truven MarketScan databases, respectively, to show significant nationwide increases in PA endoscopic procedures. ${ }^{6,35}$ The latter study showed an average annual transition change of 11\% toward ES, peaking between 2013 and 2014, ${ }^{6}$ which is encompassed by the 2010-2016 NCDB data used in this study. Our data add to this current literature as we demonstrate that academic institutions and western US regions may be adopting $\mathrm{ES}$ at a faster rate compared to nonacademic institutions and the central/eastern US regions, respectively. However, the timelines utilized from the NCDB are too narrow, and wider year differences (e.g., 2001-2010 vs 2011-2020) may elucidate better interpretable outcomes. Even though the increasing trend toward adoption of ES can be attributed to several potential factors, there remain controversies and contradictory reports regarding these differences. The differences in adoption could be attributed to academic surgeons shifting practice to $\mathrm{ES}$ at a faster pace compared to community surgeons, which will subsequently influence the future generation of training surgeons' preferred approach. Although our data suggest that practitioners of microscopic surgery at academic centers may be more prone to adopt the endoscopic technique, many reasons may play into why this is the case. Regardless of the institution type, we observed a clear 66\% increase in the use of ES from 2010 to 2016, at the expense of a $40 \%$ decrease in NES.

In our overall unmatched data, we observed lower rates of GTR, shorter LOS, decreased use of adjuvant radiotherapy, and lower short-term mortality rates for ES compared to NES. However, in our propensity score-matched analysis, we demonstrated similar rates of GTR, adjuvant radiotherapy use, and short-term mortality between the 2 approaches. Although several single-institution studies have suggested the superiority of ES in GTR, ${ }^{2,11,13,16,29}$ several meta-analyses and large cohort studies have demonstrated rates similar to our findings. $3,18,25,36,39,40$ Interestingly, although it was not evaluated in this paper, a surgeon's experience may influence GTR rates regardless of the approach,, 12 and a prior study demonstrated that surgeons using NES were more experienced than surgeons using ES in terms of number of cases performed and years in practice, although resection and quality of life outcomes were comparable. $^{25}$

Similarly, although rates of postoperative complications can be highly variable in the literature, our report of comparable rates in terms of short-term readmission or mortality is similar to those observed in other studies. ${ }^{1,4,16}$ In our propensity score-matched cohort, the only statistically significant postoperative outcome between the 2 cohorts was a decreased LOS in patients treated with ES, which is consistent throughout the literature. ${ }^{13,18,33,36}$ Although not analyzed in this study, the literature suggests that ES may also be associated with decreased costs, especially over time. ${ }^{7,14,37}$ The continuous trend toward ES accompanied with the remaining need for NES when ES is not available or preferred (e.g., surgeon preference, lack of a collaborating surgeon or endoscopic tools, insurance issues, hospital resources) requires surgical centers to be proficient in both approaches.

To our knowledge, this is the first study to explore factors predictive of receiving ES in patients with PA that included private insurance, higher income, or receiving care at an academic medical center. Use of ES at academic centers can be partly due to socioeconomic disparities ${ }^{17}$ or the multidisciplinary nature of ES requiring collaborating otolaryngologists and neurosurgeons and specialized instrumentation, in addition to the greater learning curve for adopting endoscopic procedures ${ }^{22,34}$ that may be readily developed at academic surgical centers. Singh et al. posited that certain centers may be reluctant to embrace the transition due to possible higher-than-accepted complication rates during the transition phase, ${ }^{39}$ even though multiple studies have demonstrated an apparently safe transition phase toward ES in terms of GTR and complication rates..$^{14,21,44}$ Moreover, the collaboration between neurosurgical and otolaryngology colleagues may provide additional benefits in terms of reduced complication profiles during the transition from NES to ES. ${ }^{26}$ We also observed that private insurance and higher income were independent predictors of receiving ES. Other studies have observed socioeconomic determinants influencing pituitary surgical approaches. ${ }^{28,32}$ Specifically, African American race and government insurance status have been shown to influence postoperative LOS. ${ }^{38}$ In our data, uninsured and African American patients were less likely to undergo ES, which was shown to be associated with shorter LOS.

Even though patients without insurance, those with a low income, or those receiving care at nonacademic centers are less likely to receive ES, this may not be the most overall economically sound option, because studies have shown ES to be associated with shorter hospitalization $^{13,18,33,36}$ and lower overall costs. ${ }^{14,37}$ Also, although microscopic surgery has superior stereoscopic depth perception, ES has a dynamic, wider view with the ability to work in areas that would not have been visualized otherwise, especially with the use of angled endoscopes. Because NES included open approaches in the current study, larger tumors were probably more commonly approached nonen- 
doscopically. This is interesting because prior studies have indicated that for large tumors, ES provides better rates of GTR compared to NES.,39 As such, in addition to an overall temporal trend toward ES, we observed a significant increase in the use of ES in resecting large tumors ( $\geq$ $2 \mathrm{~cm}$ ) across years. With the continuous trend toward ES and shifting of surgeons' preference and teaching focus, especially in academic centers, this may change over time. Last, we observed that the presence of medical comorbidities was a positive predictor of receiving ES. We attribute this to the possibility that ES may protect against certain postoperative complications ${ }^{18,39,40}$ and have better longterm quality of life outcomes, ${ }^{27}$ and the higher likelihood that patients with more medical complexity (i.e., comorbidities or chronic conditions) will present to academic centers. ${ }^{41}$

In comparing patients who underwent ES with those whose treatment started as ES and was converted to NES, we found that uninsured/unknown insurance status, nonCaucasian race, and shorter distance to provider were associated with conversion from ES to NES. A NCDB study of patients receiving minimally invasive radical prostatectomies observed that conversion from ES to NES was associated with African American race and low-volume centers. ${ }^{42}$ Another NCDB study by Arnold et al. of robotically assisted thoracoscopic lobectomy found that conversion from ES to NES was associated with increased shortterm mortality. ${ }^{5}$ Due to the scarcity of studies exploring this question in patients with PA, further investigations into surgical conversion are warranted.

Although we took diligent care in data collection and analysis for this study, it is important to outline several limitations that may affect such a database study by design. As previously mentioned, the NCDB only differentiated between endoscopic and "open" surgeries, the latter of which may include either microscopic or open approaches. Thus, this study focused on ES in comparison to NES as opposed to more specific variations, such as ES in comparison to microscopic transsphenoidal surgeries. The lack of differentiation between craniotomies and microscopic transsphenoidal surgeries may have contributed to the observation that larger tumors were more likely to be approached nonendoscopically, warranting continuous investigations into direct comparisons between the 3 possible approaches. Furthermore, although the specific specialties of the involved surgeons were not available in the NCDB, future studies can investigate the influence of otolaryngology cosurgeons on pituitary surgery outcomes.

Another limitation of the study consisted of the NCDB's tumor size reporting as a single $2 \mathrm{D}$ value, which encourages institutional studies to compare size-dependent outcomes based on 3D values. Furthermore, size is correlated to specific extrasellar extension (e.g., suprasellar, cavernous sinus), but this information remains elusive in the NCDB. Even though the 2004-2016 data were available in the NCDB, our analysis was limited to post-2010 cases because there were no specifications of ES or NES surgical approaches prior to that date. In addition to being limited by the collected variables due to its retrospective study design, the dataset is also limited to the selected participating hospitals, which may not accurately represent the entire population. For instance, there may exist centerto-center differences with respect to decision-making or postoperative protocols. The database nature of this study is also dependent on the accuracy of coded data. For instance, even though "endoscopic converted to open" was a specific option under surgical approach and was used by this study to analyze conversion as well as combining these cases with the NES cohort for general comparison, some clinicians may mistakenly code converted patients as ES or NES. Last, these results may suffer from safety endpoint deficiencies because certain clinical variables such as visual outcomes, endocrinopathies, CSF leaks, and other medical/surgical complications, all of which can influence treatment decision-making and outcomes, were not available in the NCDB for evaluation. Despite these limitations, this is one of the largest cohort studies to compare ES and NES resection of PA and the first to investigate clinical and demographic factors predictive of receiving ES, and we hope that the points and questions raised will lead to further investigations and improvements in surgical management of PA.

\section{Conclusions}

There is an increasing trend toward adoption of ES at the expense of NES in PA resection. Although several postoperative outcomes may be similar, ES was associated with shorter hospitalization. Factors such as tumor size, insurance status, facility type, income, race, and comorbidities may predict patients undergoing ES. In a national database, patients with smaller tumor size, Caucasian race, medical comorbidities, private insurance, higher income, more recent diagnosis, and treatment at academic centers were more likely to undergo ES for PA resection. Continuous investigation into clinical outcome measures and the rationale behind certain clinicians' preferences for ES is warranted in this complex landscape.

\section{Acknowledgments}

Dr. Abouzari and Mr. Abiri are supported by the NIH through grants TL1TR001415-04 and T32GM008620, respectively.

\section{References}

1. Agam MS, Wedemeyer MA, Wrobel B, Weiss MH, Carmichael JD, Zada G: Complications associated with microscopic and endoscopic transsphenoidal pituitary surgery: experience of 1153 consecutive cases treated at a single tertiary care pituitary center. J Neurosurg 130:1576-1583, 2019

2. Akbari H, Malek M, Ghorbani M, Ramak Hashemi SM, Khamseh ME, Zare Mehrjardi A, et al: Clinical outcomes of endoscopic versus microscopic trans-sphenoidal surgery for large pituitary adenoma. Br J Neurosurg 32:206-209, 2018

3. Almutairi RD, Muskens IS, Cote DJ, Dijkman MD, Kavouridis VK, Crocker E, et al: Gross total resection of pituitary adenomas after endoscopic vs. microscopic transsphenoidal surgery: a meta-analysis. Acta Neurochir (Wien) 160:10051021,2018

4. Ammirati M, Wei L, Ciric I: Short-term outcome of endoscopic versus microscopic pituitary adenoma surgery: a systematic review and meta-analysis. J Neurol Neurosurg Psychiatry 84:843-849, 2013

5. Arnold BN, Thomas DC, Narayan R, Blasberg JD, Detterbeck FC, Boffa DJ, et al: Robotic-assisted lobectomies in the 
National Cancer Database. J Am Coll Surg 226:1052-1062. e15, 2018

6. Asemota AO, Ishii M, Brem H, Gallia GL: Comparison of complications, trends, and costs in endoscopic vs microscopic pituitary surgery: analysis from a US health claims database. Neurosurgery 81:458-472, 2017

7. Azad TD, Lee YJ, Vail D, Veeravagu A, Hwang PH, Ratliff $\mathrm{JK}$, et al: Endoscopic vs. microscopic resection of sellar lesions - a matched analysis of clinical and socioeconomic outcomes. Front Surg 4:33, 2017

8. Boffa DJ, Rosen JE, Mallin K, Loomis A, Gay G, Palis B, et al: Using the National Cancer Database for outcomes research: a review. JAMA Oncol 3:1722-1728, 2017

9. Bokhari AR, Davies MA, Diamond T: Endoscopic transsphenoidal pituitary surgery: a single surgeon experience and the learning curve. Br J Neurosurg 27:44-49, 2013

10. Bur AM, Brant JA, Newman JG, Hatten KM, Cannady SB, Fischer JP, et al: Incidence and risk factors for prolonged hospitalization and readmission after transsphenoidal pituitary surgery. Otolaryngol Head Neck Surg 155:688-694, 2016

11. Castaño-Leon AM, Paredes I, Munarriz PM, Jiménez-Roldán L, Hilario A, Calatayud M, et al: Endoscopic transnasal trans-sphenoidal approach for pituitary adenomas: a comparison to the microscopic approach cohort by propensity score analysis. Neurosurgery 86:348-356, 2020

12. Chi F, Wang Y, Lin Y, Ge J, Qiu Y, Guo L: A learning curve of endoscopic transsphenoidal surgery for pituitary adenoma. J Craniofac Surg 24:2064-2067, 2013

13. DeKlotz TR, Chia SH, Lu W, Makambi KH, Aulisi E, Deeb Z: Meta-analysis of endoscopic versus sublabial pituitary surgery. Laryngoscope 122:511-518, 2012

14. Eseonu CI, ReFaey K, Garcia O, Salvatori R, QuinonesHinojosa A, ReFaey K, et al: Comparative cost analysis of endoscopic versus microscopic endonasal transsphenoidal surgery for pituitary adenomas. J Neurol Surg B Skull Base 79:131-138, 2018

15. Esquenazi Y, Essayed WI, Singh H, Mauer E, Ahmed M, Christos PJ, et al: Endoscopic endonasal versus microscopic transsphenoidal surgery for recurrent and/or residual pituitary adenomas. World Neurosurg 101:186-195, 2017

16. Gao Y, Zheng H, Xu S, Zheng Y, Wang Y, Jiang J, et al: Endoscopic versus microscopic approach in pituitary surgery. $\mathbf{J}$ Craniofac Surg 27:e157-e159, 2016

17. Goljo E, Parasher AK, Iloreta AM, Shrivastava R, Govindaraj S: Racial, ethnic, and socioeconomic disparities in pituitary surgery outcomes. Laryngoscope 126:808-814, 2016

18. Goudakos JK, Markou KD, Georgalas C: Endoscopic versus microscopic trans-sphenoidal pituitary surgery: a systematic review and meta-analysis. Clin Otolaryngol 36:212-220, 2011

19. Hardy J: [Excision of pituitary adenomas by trans-sphenoidal approach.] Union Med Can 91:933-945, 1962 (French)

20. Jankowski R, Auque J, Simon C, Marchal JC, Hepner H, Wayoff M: Endoscopic pituitary tumor surgery. Laryngoscope 102: 198-202, 1992

21. Karppinen A, Kivipelto L, Vehkavaara S, Ritvonen E, Tikkanen E, Kivisaari R, et al: Transition from microscopic to endoscopic transsphenoidal surgery for nonfunctional pituitary adenomas. World Neurosurg 84:48-57, 2015

22. Leach P, Abou-Zeid AH, Kearney T, Davis J, Trainer PJ, Gnanalingham KK: Endoscopic transsphenoidal pituitary surgery: evidence of an operative learning curve. Neurosurgery 67:1205-1212, 2010

23. Li A, Liu W, Cao P, Zheng Y, Bu Z, Zhou T: Endoscopic versus microscopic transsphenoidal surgery in the treatment of pituitary adenoma: a systematic review and meta-analysis. World Neurosurg 101:236-246, 2017

24. Li J, Ding W, Huang Z, Xie B, Li ZY: Comparison of shortterm outcomes between endoscopic and microscopic trans- sphenoidal surgery for the treatment of pituitary adenoma. J Craniofac Surg 30:2421-2424, 2019

25. Little AS, Kelly DF, White WL, Gardner PA, FernandezMiranda JC, Chicoine MR, et al: Results of a prospective multicenter controlled study comparing surgical outcomes of microscopic versus fully endoscopic transsphenoidal surgery for nonfunctioning pituitary adenomas: the Transsphenoidal Extent of Resection (TRANSSPHER) Study. J Neurosurg [epub ahead of print March 22, 2019. DOI: 10.3171/2018.11. JNS181238]

26. Lofrese G, Vigo V, Rigante M, Grieco DL, Maresca M, Anile $\mathrm{C}$, et al: Learning curve of endoscopic pituitary surgery: experience of a neurosurgery/ENT collaboration. J Clin Neurosci 47:299-303, 2018

27. McCoul ED, Bedrosian JC, Akselrod O, Anand VK, Schwartz TH: Preservation of multidimensional quality of life after endoscopic pituitary adenoma resection. J Neurosurg 123:813-820, 2015

28. McKee S, Yang A, Kidwai S, Govindaraj S, Shrivastava R, Iloreta A: The socioeconomic determinants for transsphenoidal pituitary surgery: a review of New York State from 1995 to 2015. Int Forum Allergy Rhinol 8:1145-1156, 2018

29. Messerer M, De Battista JC, Raverot G, Kassis S, Dubourg J, Lapras V, et al: Evidence of improved surgical outcome following endoscopy for nonfunctioning pituitary adenoma removal. Neurosurg Focus 30(4):E11, 2011

30. Molitch ME: Diagnosis and treatment of pituitary adenomas: a review. JAMA 317:516-524, 2017

31. Mortini P: Cons: endoscopic endonasal transsphenoidal pituitary surgery is not superior to microscopic transsphenoidal surgery for pituitary adenomas. Endocrine 47:415-420, 2014

32. Parasher AK, Workman AD, Kidwai SM, Goljo E, Signore $\mathrm{AD}$, Iloreta AM, et al: Costs in pituitary surgery: racial, socioeconomic, and hospital factors. J Neurol Surg B Skull Base 79:522-527, 2018

33. Penn DL, Burke WT, Laws ER: Management of non-functioning pituitary adenomas: surgery. Pituitary 21:145-153, 2018

34. Qureshi T, Chaus F, Fogg L, Dasgupta M, Straus D, Byrne RW: Learning curve for the transsphenoidal endoscopic endonasal approach to pituitary tumors. Br J Neurosurg 30:637-642, 2016

35. Rolston JD, Han SJ, Aghi MK: Nationwide shift from microscopic to endoscopic transsphenoidal pituitary surgery. Pituitary 19:248-250, 2016

36. Rotenberg B, Tam S, Ryu WH, Duggal N: Microscopic versus endoscopic pituitary surgery: a systematic review. Laryngoscope 120:1292-1297, 2010

37. Rudmik L, Starreveld YP, Vandergrift WA, Banglawala SM, Soler ZM: Cost-effectiveness of the endoscopic versus microscopic approach for pituitary adenoma resection. Laryngoscope 125:16-24, 2015

38. Sarkiss CA, Lee J, Papin JA, Geer EB, Banik R, Rucker JC, et al: Pilot study on early postoperative discharge in pituitary adenoma patients: effect of socioeconomic factors and benefit of specialized pituitary centers. J Neurol Surg B Skull Base 76:323-330, 2015

39. Singh H, Essayed WI, Cohen-Gadol A, Zada G, Schwartz TH: Resection of pituitary tumors: endoscopic versus microscopic. J Neurooncol 130:309-317, 2016

40. Strychowsky J, Nayan S, Reddy K, Farrokhyar F, Sommer D: Purely endoscopic transsphenoidal surgery versus traditional microsurgery for resection of pituitary adenomas: systematic review. J Otolaryngol Head Neck Surg 40:175-185, 2011

41. Szekendi MK, Williams MV, Carrier D, Hensley L, Thomas $\mathrm{S}$, Cerese J: The characteristics of patients frequently admitted to academic medical centers in the United States. J Hosp Med 10:563-568, 2015

42. Weiner AB, Murthy P, Richards KA, Patel SG, Eggener SE: 
Population based analysis of incidence and predictors of open conversion during minimally invasive radical prostatectomy. J Urol 193:826-831, 2015

43. White DR, Sonnenburg RE, Ewend MG, Senior BA: Safety of minimally invasive pituitary surgery (MIPS) compared with a traditional approach. Laryngoscope 114:1945-1948, 2004

44. Yang I, Wang MB, Bergsneider M: Making the transition from microsurgery to endoscopic trans-sphenoidal pituitary neurosurgery. Neurosurg Clin N Am 21:643-651, vi, 2010

45. Younus I, Gerges MM, Dobri GA, Ramakrishna R, Schwartz TH: Readmission after endoscopic transsphenoidal pituitary surgery: analysis of 584 consecutive cases. J Neurosurg [epub ahead of print September 27, 2019. DOI: 10.3171/2019.7.JNS191558]

\section{Disclosures}

Dr. Kuan is a consultant for Intersect ENT and Stryker.

\section{Author Contributions}

Conception and design: Kuan, Goshtasbi, Lehrich. Acquisition of data: Kuan, Goshtasbi, Lehrich, Abiri. Analysis and interpretation of data: Kuan, Goshtasbi, Lehrich, Abouzari, Abiri, Birkenbeuel. Drafting the article: Kuan, Goshtasbi, Lehrich, Abouzari, Abiri, Birkenbeuel. Critically revising the article: Kuan, Lan, Wang, Cadena, Hsu. Reviewed submitted version of manuscript: all authors. Approved the final version of the manuscript on behalf of all authors: Kuan. Statistical analysis: Goshtasbi, Lehrich. Study supervision: Kuan, Lan, Wang, Cadena, Hsu.

\section{Supplemental Information \\ Previous Presentations}

Portions of this work were presented at the 2020 North American Skull Base Society Annual Scientific Meeting, February 7-9, San Antonio, TX.

\section{Correspondence}

Edward C. Kuan: University of California, Irvine, CA. eckuan@ uci.edu. 